\title{
RETRACTED
}

\section{tqdm: A Fast, Extensible Progress Meter for Python and CLI}

\section{DOI: $10.21105 /$ joss. 01277}

\section{Software}

- Review ca

- Repository e

- Archive [

Submitted: 17 February 2019 Published: 17 May 2019

\section{License}

Authors of papers retain copyright and release the work under a Creative Commons Attribution $4.0 \mathrm{In-}$ ternational License (CC-BY).

\section{Casper 0 da Costa-Luis ${ }^{1}$}

1 Independent (Non-affiliated)

\section{Introduction}

tqdm is a progress bar library designed to be fast and extensible. It is written in Python, though ports in other languages are available. tqdm means progress in Arabic (taqadum $(\dot{\mathrm{G}} \overline{\mathrm{u}} \mathrm{l}, 1963)$ ) and is an abbreviation for I love you so much in Spanish (te quiero demasiado (Yahoo Answers, 2009)).

It is a common programming problem to have iterative operations where progress monitoring is desirable or advantageous. Including statements within a for loop to print out the current iteration number is a common strategy. However, there are many improvements which could be made in such a scenario:

- preventing excessive printing, such as only displaying every $n^{\text {th }}$ iteration;

- displaying iteration rate;

- displaying elapsed and estimated completion times, and

- showing all of the above on one continuously updating line.

Addressing all these issues may well take up more developer time and effort than the rest of the content of the loop. Any changes to iteration rates or attempts to re-use the printing logic in a different loop may well result in suboptimal display rates - displaying every $n^{\text {th }}$ iteration may be too (in)frequent - requiring manual adjustment of $n$ to fix.

tqdm addresses all of these problems once and for all, taking advantage of Pythonic patterns to make it a trivial task to add visually appealing, customisable progress bars without any significant performance degradation even in the most demanding of scenarios.

tqdm is intended to be used in frontends (giving end users a visual indication of progress of computations or data transfer). It is also useful for developers for debugging purposes, both as a profiling tool and also as a way of displaying logging information of an iterative task (such as error during training of machine learning algorithms). Due to its ease of use, the library is also an ideal candidate for inclusion in Python educational courses. For general (not necessarily Python) purposes, the command-line interface (CLI) mode further presents a useful tool for CLI users and system administrators monitoring data flow through pipes.

\section{Features}

Exhaustive documentation may be found on the project's home page.

The two basic use cases are within Python code and within a CLI: 


\section{Python Iterable Wrapper}

tqdm's primary (and original) use is as a wrapper around Python iterables. A simple case would be:

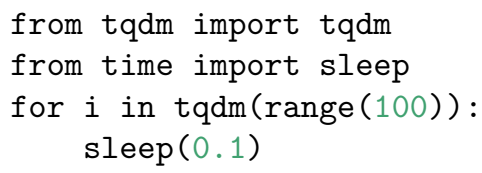

Supported features include:

- Display customisation via arguments such as desc, postfix and bar_format

- Automatic limiting of display updates to avoid slowing down due to excessive iteration rates (Stack Overflow, 2019)

- Automatic detection of console width to fill the display

- Automatic use of Unicode to render smooth-filling progress bars on supported terminals

- Support for custom rendering frontends, including:

- Command-line interface

- Jupyter HTML notebooks

- matplotlib

- Support for custom hooks/callbacks, including:

- pandas

- keras (Ben, 2019)

\section{Command-line Interface (CLI)}

A CLI is also provided, where tqdm may be used a pipe:

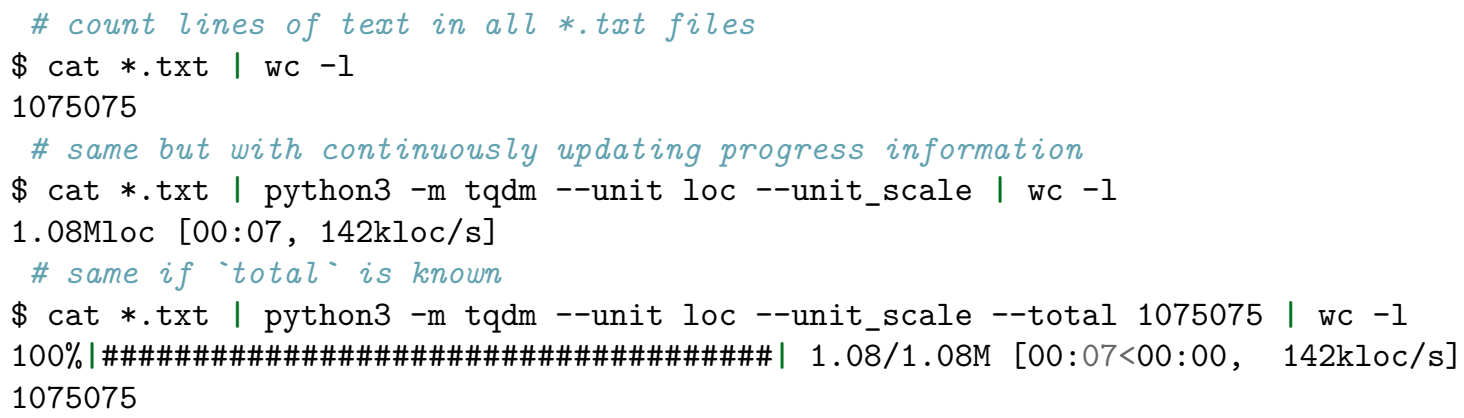

\section{Availability}

The package supports both Python versions 2 and 3, and is available for download via conda (Anaconda, 2019), pip (Python Package Index (PyPI), 2019), snap (Snapcraft, 2019), docker (Docker Inc., 2019), and Zenodo (da Costa-Luis \& tqdm developers, 2019). Web-based Jupyter interactive demonstrations are also available (Binder, 2019; Notebooks AI, 2019)

Unit tests are run at least weekly on cloud-based continuous integration (Travis CI, 2019), with code style and security issues checked on Codacy (Wikipedia, 2018). Coverage is reported on Coveralls and Codecov, and performance is monitored against regression (tqdm developers, 2019a). 


\section{Impact}

As of January 2019, tqdm has accumulated over 20 million downloads (Python Packaging Authority (PyPA), 2019), and 315 thousand code inclusions (GitHub, 2019a). Dependants of tqdm include 23 thousand repositories (GitHub, 2019b) and 7 thousand libraries (Libraries.io, 2019a). tqdm has a SourceRank of 22 (Libraries.io, 2019b), placing it in the world's top 20 Python packages as of early 2019 (Libraries.io, 2019c).

The source code of tqdm is hosted on GitHub, where it has received over 9 thousand stars (GitHub, 2019c; timqian, 2019), and was top trending repository during a period in December 2015 (Takizawa, 2018). The documentation has received over 500 thousand hits (da Costa-Luis, 2019), with highest rates during weekdays. Historical reading rates have also trended upwards at the end of holiday periods. This implies widespread use in commercial and academic settings. OpenHub valuates the work according to the constructive cost model (COCOMO) as being worth approximately $\$ 50,000$.

The library has also been used in several textbooks (Miller \& Bryce, 2017; Nandy \& Biswas, 2018; Van Boxel, 2017) and peer-reviewed scientific publications (Cook, Scholz, \& Jayawardhana, 2018; Jackson, Evangelista, Ray, \& hedrick, 2016; Knight et al., 2016; Madhikar, Åström, Westerholm, \& Karttunen, 2018; Moriwaki, Tian, Kawashita, \& Takagi, 2018; Palmer, Knight, Harper, \& Hawa, 2018; Stein, Guevarra, Newhouse, Soedarmadji, \& Gregoire, 2019). The tqdm wiki also lists other references in public media.

\section{Licence}

tqdm's source code is OSS, and all versions are archived at the DOI 10.5281/zenodo.595120. The primary maintainer Casper da Costa-Luis releases contributions under the terms of the MPLv2.0, while all other contributions are released under the terms of the MIT licence (tqdm developers, 2019b).

\section{References}

Anaconda. (2019). tqdm :: Anaconda cloud. Retrieved from https://anaconda.org/ conda-forge/tqdm

Ben. (2019). Keras integration with tqdm progress bars. Retrieved from https://github. com/bstriner/keras-tqdm

Binder. (2019). tqdm. Retrieved from https://mybinder.org/v2/gh/tqdm/tqdm/master? filepath=DEMO.ipynb

Cook, N. J., Scholz, A., \& Jayawardhana, R. (2018). Very low-mass stars and brown dwarfs in upper scorpius using gaia dr1: Mass function, disks, and kinematics. The Astronomical Journal. doi:10.3847/1538-3881/aa9751

da Costa-Luis, C. O. (2019). tqdm hits. Retrieved from https://caspersci.uk.to/cgi-bin/ hits.cgi? $q=$ tqdm\&a $=$ plot

da Costa-Luis, C. O., \& tqdm developers. (2019). tqdm stable. doi:10.5281/zenodo. 595120

Docker Inc. (2019). tqdm/tqdm - docker hub. Retrieved from https://hub.docker.com/ $\mathrm{r} / \mathrm{tqdm} / \mathrm{tqdm}$ 
GitHub. (2019a). tqdm code results. Retrieved from https://github.com/search?q= tqdm\&type $=$ Code

GitHub. (2019b). tqdm dependents. Retrieved from https://github.com/tqdm/tqdm/ network/dependents

GitHub. (2019c). tqdm stargazers. Retrieved from https://github.com/tqdm/tqdm/ stargazers

Ġūl, M. A. (1963). Early southern arabian languages and classical arabic sources: A critical examination of literary and lexicographical sources by comparison with the inscriptions (PhD thesis). SOAS University of London.

Jackson, B. E., Evangelista, D. J., Ray, D. D., \& hedrick, T. L. (2016). 3D for the people: Multi-camera motion capture in the field with consumer-grade cameras and open source software. Biology open, 5(9), 1334-1342. doi:10.1242/bio.018713

Knight, V., Campbell, O., Harper, M., Langner, K., Campbell, J., Campbell, T., Carney, A., et al. (2016). An open reproducible framework for the study of the iterated prisoner's dilemma. Journal of Open Research Software. doi:10.5334/jors.125

Libraries.io. (2019a). tqdm on PyPI. Retrieved from https://libraries.io/pypi/tqdm

Libraries.io. (2019b). SourceRank breakdown for tqdm. Retrieved from https://libraries. io/pypi/tqdm/sourcerank

Libraries.io. (2019c). Libraries - the open source discovery service. Retrieved from https://libraries.io/search?order=desc\&platforms=PyPI\&sort=rank

Madhikar, P., Åström, J., Westerholm, J., \& Karttunen, M. (2018). CellSim3D: GPU accelerated software for simulations of cellular growth and division in three dimensions. Computer Physics Communications. doi:10.1016/j.cpc.2018.05.024

Miller, P., \& Bryce, C. (2017). Python digital forensics cookbook: Effective python recipes for digital investigations. Packt Publishing Ltd.

Moriwaki, H., Tian, Y.-S., Kawashita, N., \& Takagi, T. (2018). Mordred: A molecular descriptor calculator. Journal of cheminformatics, 10(1), 4. doi:10.1186/s13321-018-0258-y

Nandy, A., \& Biswas, M. (2018). Reinforcement learning with keras, tensorflow, and chainerrl. In Reinforcement learning : With open ai, tensorflow and keras using python (pp. 129-153). Apress. doi:10.1007/978-1-4842-3285-9_5

Notebooks AI. (2019). tqdm. Retrieved from https://notebooks.ai/demo/gh/tqdm/tqdm

Palmer, G. I., Knight, V. A., Harper, P. R., \& Hawa, A. L. (2018). Ciw: An opensource discrete event simulation library. Journal of Simulation. doi:10.1080/17477778. 2018.1473909

Python Package Index (PyPI). (2019). tqdm. Python Software Foundation. Retrieved from https://pypi.org/project/tqdm/

Python Packaging Authority (PyPA). (2019). Analyzing PyPI package downloads python packaging user guide. Python Software Foundation. Retrieved from https:// packaging.python.org/guides/analyzing-pypi-package-downloads /

Snapcraft. (2019). Installing tqdm for linux using the snap store. Retrieved from https: //snapcraft.io/tqdm

Stack Overflow. (2019). Why is printing to stdout so slow? Can it be sped up? Retrieved from https://stackoverflow.com/questions/3857052/why-is-printing-to-stdout-so-slow-can-it-be-s 
Stein, H. S., Guevarra, D., Newhouse, P. F., Soedarmadji, E., \& Gregoire, J. M. (2019). Machine learning of optical properties of materials - predicting spectra from images and images from spectra. Chemical Science. doi:10.1039/C8SC03077D

Takizawa, N. (2018 19). GitHub trending history. Retrieved from https://github.com/ nihey/trending-history/blob/master/histories/Python.md

timqian. (2019). Star history. Retrieved from https://timqian.com/star-history/\#tqdm/ tqdm

tqdm developers. (2019a). Airspeed velocity. Retrieved from https://tqdm.github.io/ tqdm/

tqdm developers. (2019b). tqdm licence. GitHub. Retrieved from https://github.com/ tqdm/tqdm/blob/master/LICENCE

Travis CI. (2019). Tqdm/tqdm build status. Retrieved from https://travis-ci.org/tqdm/ tqdm

Van Boxel, D. (2017). Hands-on deep learning with tensorflow. Packt Publishing.

Wikipedia. (2018). List of tools for code review. Retrieved from https://en.wikipedia. org/wiki/List_of_tools_for_code_review

Yahoo Answers. (2009). ¿Lenguaje sms que significa esto? Retrieved from https://es. answers.yahoo.com/question/index?qid=20090405052137AAF2YBo\&guccounter=1 